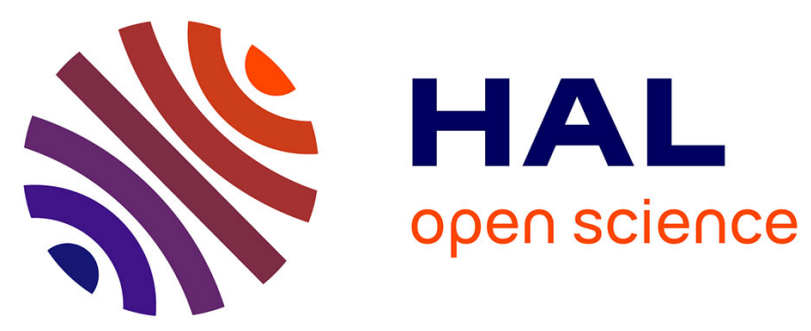

\title{
Excitation of nitrogen molecular ions in a strong laser field by electron recollisions
}

Vladimir T. Tikhonchuk, Jean-Félix Tremblay-Bugeaud, Yi Liu, Aurélien Houard, André Mysyrowicz

\section{- To cite this version:}

Vladimir T. Tikhonchuk, Jean-Félix Tremblay-Bugeaud, Yi Liu, Aurélien Houard, André Mysyrowicz. Excitation of nitrogen molecular ions in a strong laser field by electron recollisions. The European Physical Journal D: Atomic, molecular, optical and plasma physics, 2017, 71 (11), 10.1140/epjd/e2017-80464-3 . hal-01648046

\section{HAL Id: hal-01648046 \\ https://hal-ensta-paris.archives-ouvertes.fr/hal-01648046}

Submitted on 27 Nov 2017

HAL is a multi-disciplinary open access archive for the deposit and dissemination of scientific research documents, whether they are published or not. The documents may come from teaching and research institutions in France or abroad, or from public or private research centers.
L'archive ouverte pluridisciplinaire HAL, est destinée au dépôt et à la diffusion de documents scientifiques de niveau recherche, publiés ou non, émanant des établissements d'enseignement et de recherche français ou étrangers, des laboratoires publics ou privés. 


\title{
Excitation of nitrogen molecular ions in a strong laser field by electron recollisions
}

\author{
Vladimir T. Tikhonchuk ${ }^{1, a}$, Jean-Félix Tremblay-Bugeaud ${ }^{2}$, Yi Liu ${ }^{3}$, Aurélien Houard ${ }^{4}$, \\ and André Mysyrowicz ${ }^{4}$ \\ ${ }^{1}$ Centre Lasers Intenses et Applications, University of Bordeaux-CNRS-CEA, 33405 Talence, France \\ ${ }^{2}$ University Laval, Quebec, QC GV 0A6, Canada \\ 3 Shanghai Key Lab of Modern Optical System, University of Shanghai for Science and Technology, 200093 \\ Shanghai, P.R. China \\ ${ }^{4}$ Laboratoire d'Optique Appliquée, ENSTA ParisTech, CNRS, Ecole Polytechnique, University Paris-Saclay, 91762 \\ Palaiseau, France
}

\begin{abstract}
A semiclassical model of electron rescattering in a strong laser field is applied to the evaluation of the probability of nitrogen molecular ion excitation. Depending on the ionization phase, a group of electrons oscillate around the parent ion for several laser periods and rescatter at large angles. The effect of ion attraction enhances significantly the probability of ion excitation by electron-ion collisions. The total probability of ion transfer from ground to excited ionic molecular state and the corresponding dipolar moment are evaluated for typical laser filament parameters.
\end{abstract}

\section{Introduction}

It is well known that if a gas of nitrogen molecules (or air) is illuminated by an intense ultrashort laser pulse at $800 \mathrm{~nm}$, a UV luminescence appears in the form of a group of narrow lines located around the wavelength of $390 \mathrm{~nm}$. Some of these lines correspond to transitions between different vibrational levels of the excited state $\mathrm{B}^{2} \Sigma_{u}^{+}$and the ground state $\mathrm{X}^{2} \Sigma_{q}^{+}$of the singly ionized molecule. Their appearance is usually interpreted as being due to direct multiphoton excitation from the molecular neutral ground state $\mathrm{X}^{1} \Sigma_{g}^{+}$to ionic states $\mathrm{X}^{2} \Sigma_{g}^{+}$and $\mathrm{B}^{2} \Sigma_{u}^{+}$with most of the final population in $\mathrm{X}^{2} \Sigma_{g}^{+}$. However such an interpretation cannot explain a strong optical amplification for the $\mathrm{B} \rightarrow \mathrm{X}$ transition at lines 391 and $428 \mathrm{~nm}$ (corresponding to the vibration transitions $0 \rightarrow 0$ and $0 \rightarrow 1$, respectively) at sufficiently high pump pulse intensity, mostly in the direction of the pump pulse [1-5]. Two hypothesis have been proposed to solve this problem: one is based on an electron recollision model [5], the other takes into account the role of intermediate resonant state $\mathrm{A}^{2} \Pi_{u}$ of the nitrogen molecular ion to bring a population inversion [6]. In this manuscript we examine further the recollision model and concentrate on the discussion of results obtained under conditions of pump laser filamentation.

The hypothesis of electron recollisions is motivated by the dependence of the forward coherent $391 \mathrm{~nm}$ signal on

\footnotetext{
${ }^{a}$ e-mail: tikhonchuk@u-bordeaux.fr
}

the pump laser polarization [5]. Optical amplification disappears rapidly when the pump pulse ellipticity increases beyond 0.2. Such an effect can be explained by a recollision model. In the case of elliptic or circular polarization, free electrons created by tunnel ionization of nitrogen molecules never come back to the parent ions. They are moving away taking a certain amount of energy from the laser field. In contrast, in the case of a linear polarization, electrons moving in the oscillating laser field may return to the parent ions [7]. These electron recollisions are responsible for high harmonic generation, nonsequential double ionization and other atomic processes $[8,9]$. They affect also the rate of free electron heating in a plasma in strong electromagnetic fields [10-12]. In this case, the ion attraction of the oscillating electron - the so called Coulomb focusing effect [13] - increases the probability of a close approach and thus the quantity of energy transferred from the laser field to the electrons. The amplification of the line corresponding to the $\mathrm{B} \rightarrow \mathrm{X}$ transition observed in the case of a linear pump laser polarization together with the fact that the luminescence does not change significantly between linear and circular polarizations indicates the important role of electron recollisions in the optical amplification process. Another confirmation of importance of the recollision process for the coherent amplification of the nitrogen ion emission has been obtained recently in reference [14]. A strong dependence of the forward emission signal on the laser wavelength and pulse duration is explained by an interference of dipolar moments of nitrogen ions excited by electron recollisions. 
A simple one-dimensional model of recollision excitation of a molecular nitrogen ion was considered in references [5,14]. An electron is liberated by a tunnel process during the laser pulse and is moving in the oscillating laser electric field. For an electron returning to the parent ion, the effect of ion attraction was neglected and the excitation probability was introduced empirically. Although such an approach allows describing reasonably well the dynamics of electron motion, it cannot evaluate correctly the excitation probability, because it does not account for the quantum divergence of the electron cloud as it moves in the laser field, and for the attraction of the parent ion. Motivated by the observations described in references $[5,14]$, we develop here a three-dimensional semiclassical model of the ion excitation in the laser field. It assumes a quantum probability of ionization of the nitrogen molecule, a classical electron motion in the combined electric field of the laser and the electrostatic field of the ion, and an ion excitation using the experimentally measured total excitation cross section [15]. The quantum divergence of the electron cloud is modeled by a divergent cone of electron trajectories distributed according to the Gaussian function with an angular width defined by the quantum-mechanical process of tunnel ionization. The ion excitation probability in the recollision process and the average dipolar moment are defined and their dependence on the laser field intensity, pulse duration, wavelength and chirp is analyzed.

\section{Model description}

\subsection{Collisional excitation of the nitrogen ion}

We consider a semiclassical model of the electron recollision with the parent ion. In each elementary act, an electron in the presence of an intense laser field is removed from the outer orbital of the neutral nitrogen molecule and is driven back and forth by the laser field around the parent molecular ion. There is a possibility that it collides inelastically with an inner orbital electron. During this event, the inner orbital electron in the ground state $\mathrm{X}^{2} \Sigma_{g}^{+}$can transferred to the outer orbital $\mathrm{B}^{2} \Sigma_{u}^{+}$of the molecular ion. There are specific characteristics for this type of transfer. First, it can only occur if the energy of the incident electron $\varepsilon_{e}$ is larger than the excitation energy $\varepsilon_{B X} \simeq 3.17 \mathrm{eV}$. This condition is easily met in experiments [4]. Inside the laser filaments formed with a converging pump beam [16,17], the intensity reaches $I_{\text {las }} \simeq 1.5 \times 10^{14} \mathrm{~W} / \mathrm{cm}^{2}$. This corresponds to an average electron quiver energy $\simeq 9 \mathrm{eV}$ for the laser wavelength $\lambda_{\text {las }} \simeq 800 \mathrm{~nm}$, which is three times larger than the excitation energy. The second condition for the ion excitation is that the electron approaches sufficiently close the ion so that the effective impact parameter is within the radius defined by the corresponding cross section.

Instead of calculating the ion excitation cross section analytically, we chose to refer to the direct experimental measurements [15]. It is found that the cross section achieves its maximum value of $\sigma_{B X \text { max }} \simeq 1.4 \times 10^{-16} \mathrm{~cm}^{2}$ at the excitation threshold $\varepsilon_{B X}$ and slowly decreases as the electron energy $\varepsilon_{e}$ increases. In numerical simulations we use an interpolation expression for the excitation cross section, which in atomic units reads:

$$
\sigma_{B X}\left(\varepsilon_{e}\right)=1.32 \varepsilon_{e}^{-0.66} H\left(\varepsilon_{e}-\varepsilon_{B X}\right),
$$

where $H$ is the Heaviside step function. This cross section was however measured in interaction of a beam of monoenergetic electrons with a beam of molecular nitrogen ions, where the electron dynamics is controlled by the Coulomb electric field only. The electron motion in our case is dominated by the laser field with the exception of a short part of trajectory near the ion. So, in order to simplify the problem but retain the total measured cross section, we assume that the excitation takes place if the electron passes the ion within a circle of surface equal to the cross section (1). That is, the radius of this circle - the maximum excitation distance - is defined as $r_{B X}=\left[\sigma_{B X}\left(\varepsilon_{e}\right) / \pi\right]^{1 / 2}$. This definition leads to a slight underestimation of the excitation probability because the electron energy increases as it approaches the ion.

Such a classical representation of inelastic electron collisions is simpler than the reconstruction of the electron density in the vicinity of ion proposed in reference [18] for studies of high order harmonic generation, and it has the advantage of accounting for multiple returns of the same electron to the parent ion. A good agreement of a simplified semiclassical approach with a direct solution of the time-dependent Schrödinger equation was demonstrated in reference [12] in the case of electron-ion collisions in a strong laser field in a plasma.

\subsection{Electron dynamics in the laser field}

The free electron dynamics in the laser field and the field of the parent ion is described by Newton's equations. The electric field of ion, $Z e \boldsymbol{r}_{e} / 4 \pi \epsilon_{0} r_{e}^{3}$, where, $Z e$ is the ion charge, $\epsilon_{0}$ is the vacuum dielectric permittivity and $\boldsymbol{r}_{e}$ is the electron coordinate with respect to ion, is approximated by a central Coulomb electrostatic force. As the nitrogen molecular orbital has a bonding symmetry, its ionization probability is close to the ionization probability of an argon atom having the same ionization potential [19]. Therefore the contribution of the dipolar component of the ion field to the electron orbit is relatively small.

The laser field is linearly polarized with an electric field $\boldsymbol{E}=(E, 0,0)$ and magnetic field $\boldsymbol{B}=(0, E / c, 0)$, where $E(t)=E_{0}(t) \cos \varphi(t)$. The amplitude $E_{0}(t)$ corresponds to a flat-top envelope, $E_{0}(t)=E_{m} \exp \left[-\left(2 t / t_{\text {las }}-1\right)^{12}\right]$ with maximum amplitude $E_{m}$, steep edges and pulse duration $t_{\text {las }}$. This choice of the envelope is motivated by the nonlinear clamping of laser pulse intensity in the filament [17]. The phase $\varphi(t)=\omega_{0} t+(C / 4)\left(t / t_{\mathrm{las}}-1\right)^{2}$ describes a chirped pulse with carrier frequency $\omega_{0}$, pulse duration $t_{\text {las }}=t_{0 \text { las }}\left(C^{2}+1\right)^{1 / 2}$ and $\operatorname{chirp} C$.

The initial conditions for the electron velocity $v_{e x}\left(t_{0}\right)=$ $0, v_{e \perp}\left(t_{0}\right)=v_{0 \perp}$, and coordinate $x_{e}\left(t_{0}\right)=x_{0}$ and $y_{e}\left(t_{0}\right)=$ $z_{e}\left(t_{0}\right)=0$ at the moment $t_{0}$ of nitrogen molecule ionization are defined as follows. The electron released from the atom is placed on axis $x$ at distance $x_{0}$ opposite to the electric field direction. This distance is found from the 
tunnel condition, that is, the free electron energy in the laser and ion potential is the same as in the bound state:

$$
-e E\left(t_{0}\right) x_{0}-\frac{e^{2}}{4 \pi \epsilon_{0}\left|x_{0}\right|}=-U_{i}
$$

where $U_{i}=15.576 \mathrm{eV}$ is the binding energy of the electron in the nitrogen molecule. Solving this quadratic equation one finds an explicit expression for $x_{0}$. In atomic units it reads:

$$
\begin{aligned}
x_{0} & =-\left(U_{i}+\sqrt{U_{i}^{2}-4 E\left(t_{0}\right)}\right) / 2 E\left(t_{0}\right) . \\
& \approx-U_{i} / E\left(t_{0}\right) .
\end{aligned}
$$

We recall here the normalization factors: $E_{\text {au }}=e / 4 \pi \epsilon_{0} a_{B}^{2}$ $=5.14 \times 10^{11} \mathrm{~V} / \mathrm{m}$ is the atomic electric field, $\omega_{\mathrm{au}}=$ $e E_{a u} a_{B} / \hbar=41 \mathrm{fs}^{-1}$ is the inverse atomic time, $a_{B}=$ $4 \pi \epsilon_{0} \hbar^{2} / m_{e} e^{2}=0.0529 \mathrm{~nm}$ is the Bohr radius, and $m_{e}$ is the electron mass. In practice, for a laser intensity $I_{\text {las }}=1.5 \times 10^{14} \mathrm{~W} / \mathrm{cm}^{2}$ at wavelength $\lambda_{\text {las }}=800 \mathrm{~nm}$, the laser field amplitude is $\simeq 0.066$ a.u., the laser period $T=2 \pi c / \lambda_{\text {las }} \simeq 110$ a.u., and the typical distance of electron release is about $4-8$ a.u. The latter is sufficient for the electron initial dynamics to be dominated by the laser electric force, but it is about 10 times smaller than the typical electron excursion length in the laser field. The initial electron velocity in the electric field direction is set to zero, as it in any case much smaller than the one gained from the laser. In contrast, the transverse velocity is chosen within the probability distribution defined according to references $[20,21]$ by a Gaussian function

$$
p_{v}\left(v_{0 \perp}\right)=\frac{1}{\left\langle v_{\perp}\right\rangle^{2}} \exp \left(-\frac{v_{0 \perp}^{2}}{2\left\langle v_{\perp}\right\rangle^{2}}\right) .
$$

These initial conditions are very similar to those used in reference [13] for modeling the double ionization of helium. The expression for the characteristic dispersion velocity $\left\langle v_{\perp}\right\rangle$ is given by equation (8) in Section 2.3.

The electron orbit has been calculated by solving the dynamic equations. For the numerical integration they have been presented in the atomic units as follows:

$$
\begin{aligned}
\frac{d^{2} x_{e}}{d t^{2}} & =-E(t)\left(1-\alpha \frac{d z_{e}}{d t}\right)-\frac{x_{e}}{\left(r_{e}^{2}+\epsilon^{2}\right)^{3 / 2}}, \\
\frac{d^{2} y_{e}}{d t^{2}} & =-\frac{y_{e}}{\left(r_{e}^{2}+\epsilon^{2}\right)^{3 / 2}}, \\
\frac{d^{2} z_{e}}{d t^{2}} & =-\alpha E(t) \frac{d x_{e}}{d t}-\frac{z_{e}}{\left(r_{e}^{2}+\epsilon^{2}\right)^{3 / 2}},
\end{aligned}
$$

where $Z=1, r_{e}=\left(x_{e}^{2}+y_{e}^{2}+z_{e}^{2}\right)^{1 / 2}, \alpha=e^{2} / 4 \pi \epsilon_{0} \hbar c=$ $1 / 137$ is the fine structure constant and $\epsilon \simeq 0.05$ is a small factor softening the Coulomb potential at small distances. These equations were solved numerically using the "Mathematica" or "Python" packages starting from time $t_{0}$. As soon as the dynamic equations were solved numerically, the electron trajectory $\boldsymbol{r}_{e}(t)$ and electron energy $\varepsilon_{e}=\frac{1}{2} m_{e} \boldsymbol{v}_{e}^{2}$ were analyzed according to the ion excitation criterion. If along the trajectory the condition $r_{e}(t) \leq r_{X B}\left(\varepsilon_{e}(t)\right)$ was met and the electron energy was larger than the excitation energy, the corresponding time $t_{s}$ is recorded and the ion considered to be excited.

A typical electron orbit is shown in Figure 1a. An electron is born at the position $x_{0} \approx-5$, it moves in the electric field to distance $x \sim-20$ and then returns back to the ion. While passing close by, it is attracted by the Coulomb force and briskly changes its direction. In this particular case the excitation condition was satisfied and the ion is assumed to be excited. The zoom in Figure 1b of the recollision event shows the dominant role of the Coulomb force as the electron-ion distance at that time is comparable to the size of ion. This is confirmed in Figure 1c showing the time dependence of the electron-ion distance and the electron kinetic energy. In particular, the energy is more than 2 a.u., which exceeds the laser ponderomotive potential more than 5 times. The increase of the electron energy at the moment of collision from 1.5 to 2.5 a.u. is due to the Coulomb attraction.

The ion Coulomb force strongly deviates the electron orbit so it either rejected far from the ion or can be trapped temporarily in a close orbit for a few cycles, but it is eventually liberated by the electric field and is ejected in some arbitrary direction. The role of the laser magnetic field is very minor. Neglecting the magnetic field component in equation (4) (by formally setting $\alpha=0$ ) results in an axial symmetry around the $x$-axis and in a planar trajectory in the plane defined by the laser polarization ( $x$-axis) and the direction of initial transverse velocity. Accounting for the magnetic field component leads to a small deviation of the symmetry axis in the $x, z$-plane, which has no significant effect on the recollision probability. For this reason and also for saving simulation time, the calculations were conducted in the planar geometry and the results were averaged over the electron initial distribution assuming the axial symmetry.

The duration of each run was chosen empirically to correspond to the time when the electron finds itself far away from the ion. In practice, the electron passes several times near the parent ion before going away. The ion excitation is possible at any crossing provided the electron-ion distance satisfies the excitation criterion, but the probability decreases with time. About half of the recollision excitations were detected within one period after the electron release. They correspond to electrons launched with a very small transverse velocity and at times a little after the laser field maximum. The electrons launched before the laser field maximum and with larger transverse velocities may enter in the recollision at subsequent crossings or leave the ion without recollision.

\subsection{Ionization of the nitrogen molecule in a laser field}

The atom ionization rate in an oscillating electric field $E(t)$ was calculated by Perelomov et al. [20] and discussed in detail in review [21]. There are two limits depending on the value of the Keldysh parameter

$$
\gamma(E)=\left(\omega_{0} / e|E|\right)\left(2 m_{e} U_{i}\right)^{1 / 2} .
$$




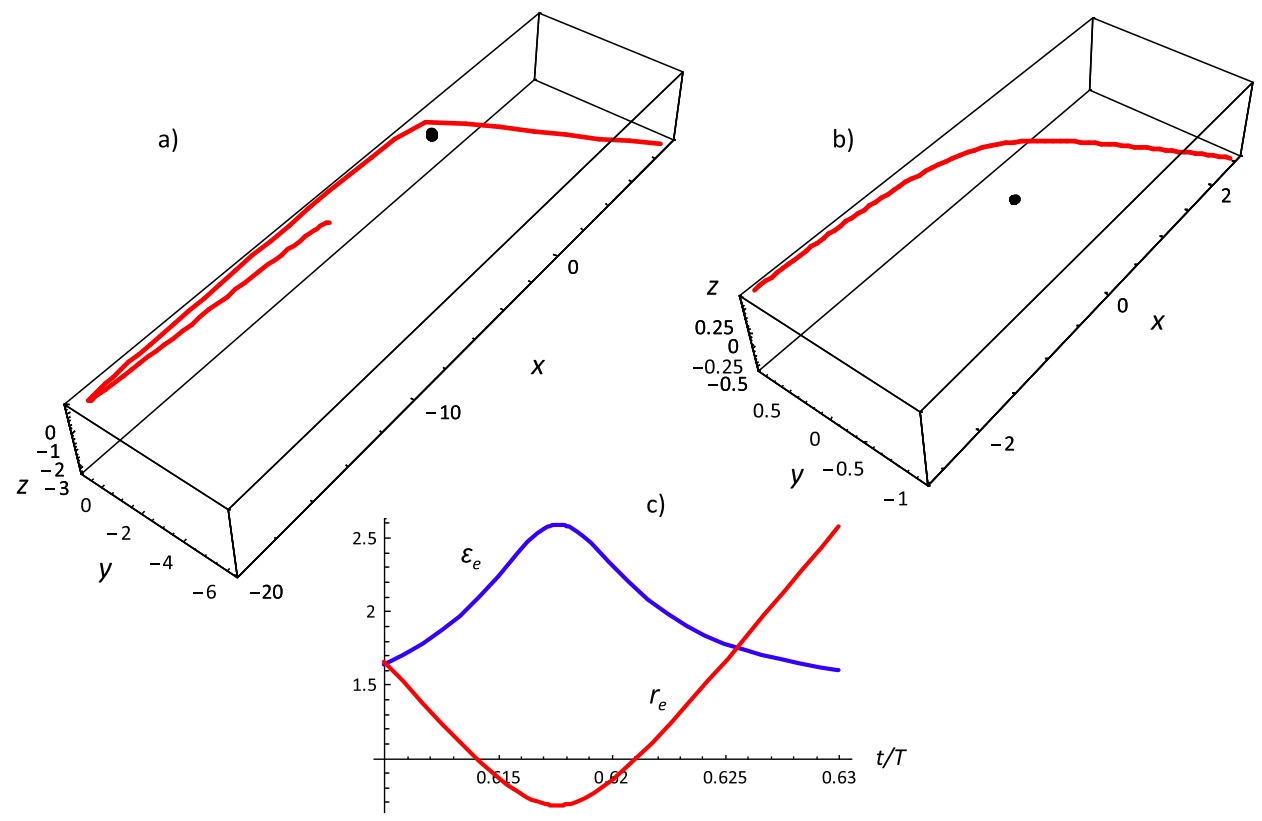

Fig. 1. Typical three dimensional trajectory (red) of the electron. The black dot shows the position of the ion. (a) General view of the trajectory from the born time $t_{0}$ and past the recollision time. (b) Enlarged view near the recollision event during the time of 3 a.u. (c) Distance electron-ion (red) and electron kinetic energy (blue) during the recollision event in atomic units.

The limit $\gamma \gg 1$ corresponds to the multiphoton ionization, contrary to the tunnel ionization in the opposite limit. The PPT model [20] provides quite accurate expressions for the ionization rate in both limits [22]. Moreover, it applies in the intermediate case $\gamma \sim 1$, which corresponds to the set parameters we are interested in. A detailed comparison of strong field ionization of diatomic molecules with their companion atoms having nearly equal ionization potentials $[23,24]$ shows a very small difference in the case of the nitrogen molecule. The ionization rate from level with orbital quantum number $l=0$ is written as follows:

$$
\begin{aligned}
w_{\text {ion }}(E)= & \omega_{a u} \sqrt{\frac{3}{2 \pi}} \kappa^{2} C_{k}^{2}\left(\frac{2 \kappa^{3} E_{a u}}{|E|}\right)^{2 n^{*}-3 / 2} \\
& \times \exp \left(-\frac{2 \kappa^{3} E_{a u}}{3|E|} g(\gamma)\right)
\end{aligned}
$$

where $\kappa=\sqrt{2 U_{i} / \hbar \omega_{a u}}, C_{k}=2^{n^{*}} / \Gamma\left(n^{*}+1\right), n^{*}=Z / \kappa$, and

$$
g(\gamma)=(3 / 2 \gamma)\left[\left(1+1 / 2 \gamma^{2}\right) \operatorname{arcsinh} \gamma-\sqrt{1+\gamma^{2}} / 2 \gamma\right]
$$

The lowest ionization potential $U_{i}=15.576 \mathrm{eV}$ of nitrogen molecule corresponds to the $3 \sigma_{g}$ orbital [25]. Using expression (6), one can evaluate the ionization probability per half laser period,

$$
W_{k \text { ion }}=\int_{k T / 2}^{(k+1) T / 2} d t w_{\text {ion }}(E(t))
$$

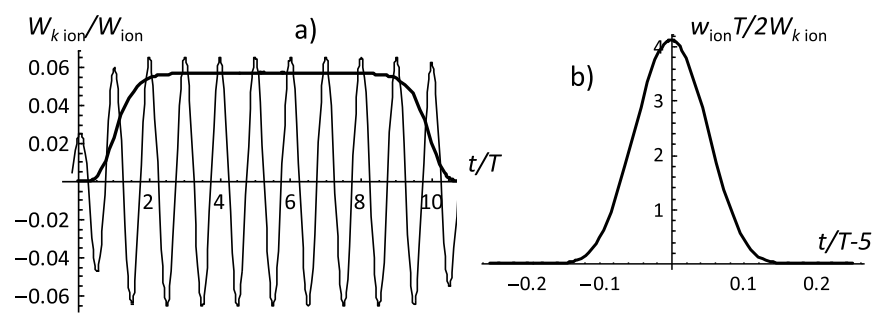

Fig. 2. (a) Dependence of the nitrogen ionization probability over a half laser period $W_{k \text { ion }} / W_{\text {ion }}$ (thick line) on time. It is normalized to the total ionization probability (7). Gray line shows the time dependence of the laser field in arbitrary units. (b) Time dependence of the nitrogen ionization rate within a half of laser cycle $k=10$ normalized to the ionization probability over a half of laser cycle $w_{\text {ion }}(E(t)) T / 2 W_{k}$ ion.

where $k$ is an integer number and $T=2 \pi / \omega_{0}$ is the laser period. Consequently, the total ionization probability is calculated as a sum over the entire laser pulse duration:

$$
W_{\text {ion }}=\sum_{1}^{M} W_{k \text { ion }},
$$

where $M=\left[2 t_{\text {las }} / T\right]$ is the total number of laser half periods. It is assumed that the pulse duration is sufficiently short so that the fraction of ionized molecules is small, $W_{\text {ion }} \ll 1$.

Figure 2a shows the ionization probability $W_{k}$ ion of the molecular nitrogen over a half laser period for a laser maximum intensity $1.5 \times 10^{14} \mathrm{~W} / \mathrm{cm}^{2}$, a wavelength $800 \mathrm{~nm}$ and a pulse duration of $30 \mathrm{fs}$. It is normalized to the total 

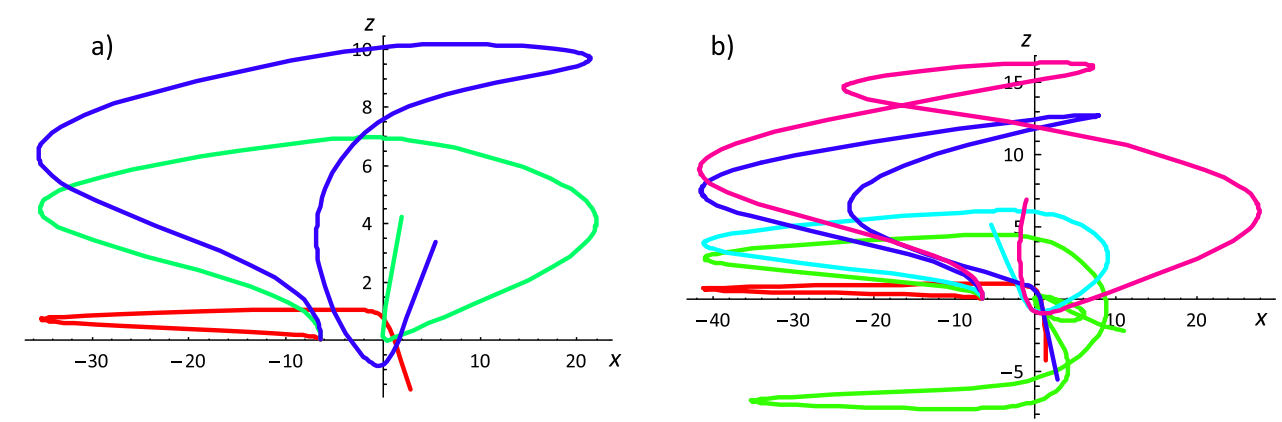

Fig. 3. Examples of electron orbits in the $x, z$ plane (in a.u.) leading to the recollision and ion excitation. The laser intensity is $1.5 \times 10^{14} \mathrm{~W} / \mathrm{cm}^{2}$, the wavelength $800 \mathrm{~nm}$. (a) The ionization time is $t_{0}=5.99 \mathrm{~T}$, that is $0.01 \mathrm{~T}$ before the maximum of the laser field, the initial transverse velocities are 0.017 (red), 0.110 (green) and 0.155 (blue) a.u. (b) The ionization time is $0.025 T$ before the maximum of the laser field, the initial transverse velocities are 0.015 (red), 0.0638 (green), 0.087 (light blue), 0.168 (blue) and 0.200 (mauve) a.u. The electric field at $t_{0}$ is directed in the positive direction of the $x$-axis.

ionization probability $W_{\text {ion }}$, which is $2.6 \%$ in this particular case. The ionization process takes place within 9 laser periods, which is comparable with the laser pulse duration. The ionization process is tightly localized near the laser electric field extrema. According to Figure 2b, the ionization takes place over $20 \%$ of the period near each maximum. Only these time intervals are interesting for studies of the recollision process. In numerical simulations we extended this time interval to $25 \%$ around each field extremum for completeness.

The ionization in the ion ground state $\mathrm{X}^{2} \Sigma_{g}^{+}$can be compared with the field ionization in the ion excited state $\mathrm{B}^{2} \Sigma_{u}^{+}$. The binding energy of an electron in the nitrogen molecule at the orbital $2 \sigma_{u}$ is $U_{i}=18.746 \mathrm{eV}$. The probability of ejection of that electron is approximately two orders of magnitude smaller than the electron at the higher orbital $3 \sigma_{g}$. That is, the laser field ionization creates about $1.5 \%$ nitrogen ions in the excited state for a laser intensity $1.5 \times 10^{14} \mathrm{~W} / \mathrm{cm}^{2}$ and a pulse duration of $30 \mathrm{fs}$. This number increases to $2.5 \%$ in the case of a laser intensity of $2 \times 10^{14} \mathrm{~W} / \mathrm{cm}^{2}$.

According to references $[20,21]$, the ionization probability is maximal for an electron released with zero kinetic energy. The dependence of the ionization probability on the component of electron velocity perpendicular to the electric field is given by equation (3), where the characteristic velocity reads:

$$
\left\langle v_{\perp}\right\rangle=\left(\kappa \hbar \omega_{0} / 2 m_{e} \operatorname{arcsinh} \gamma\right)^{1 / 2}
$$

For our parameters of interest the characteristic value of $\left\langle v_{\perp}\right\rangle$ is $\sim 0.2$ a.u.

\section{Simulation results}

\subsection{Average over the transverse initial velocities}

Quantum divergence of the electron cloud is an important part of the recollision process. It is modeled with a bunch of electron trajectories generated at the same ionization time with the same initial conditions except for the transverse velocity, which is varied according to quantum probability (3). It was found in test runs that electrons liberated with transverse velocities $v_{0 \perp}>\left\langle v_{\perp}\right\rangle$ never come back close to the parent ion. For that reason in simulations we limited $v_{0 \perp}$ by the cutoff of $v_{\max \perp}=0.25 \gtrsim\left\langle v_{\perp}\right\rangle$ and used $N_{v} \simeq 500$ trial electrons with the velocities $v_{\perp n}$ homogeneously distributed over the interval from 0 to $v_{\max \perp}$ with the step $\Delta v_{\perp}=v_{\max \perp} / N_{v}$.

The collision time $t_{s}$ has been recorded in each run where the electron trajectory verified the ion excitation conditions $r_{e}(t) \leq r_{B X}\left(\varepsilon_{e}(t)\right)$. If electrons would follow similar orbits, time $t_{s}$ would be a smooth function of the initial velocity, but it is not the case. As it is shown in Figure 3, there are several possible orbits corresponding to quite different collision times depending on the ionization time and initial velocity. The electrons released with a very small transverse velocity $v_{\perp}<0.03-0.05$ a.u. have a large probability to return to the ion within one laser period. The red lines in panels (a) and (b) show such trajectories. However, such electrons represent a relatively small fraction of the whole distribution (3), and their contribution to the excitation probability is relatively small. The electrons released with larger transverse velocities do not return to the ion in the first laser period, but they are passing nearby and their trajectory is influenced by ion attraction. These electrons have a large probability to approach the ion after two, three or even four returns (see green and blue curves in Fig. 3). Such orbits are observed for the electrons released a short time before the maximum of the laser field. These electrons are making the most significant contribution to the total excitation probability.

An example of electron recollision characteristics for a given launch time is shown in Figure 4. The recollision time is constant and equal approximately to the laser period for small perpendicular velocities ranging from 0 to 0.03 a.u. The electron energy at the recollision moment is increasing enormously because of the Coulomb attraction as the electron passes close to the ion. The recollision dynamics becomes more complicated for larger initial transverse velocities. There are groups of trajectories corresponding to different recollision times, as it is shown in Figure 3, corresponding to rather high electron energies. The recollision time increases with the initial electron velocity, achieving values of about three laser periods for large $v_{0 \perp}$. 

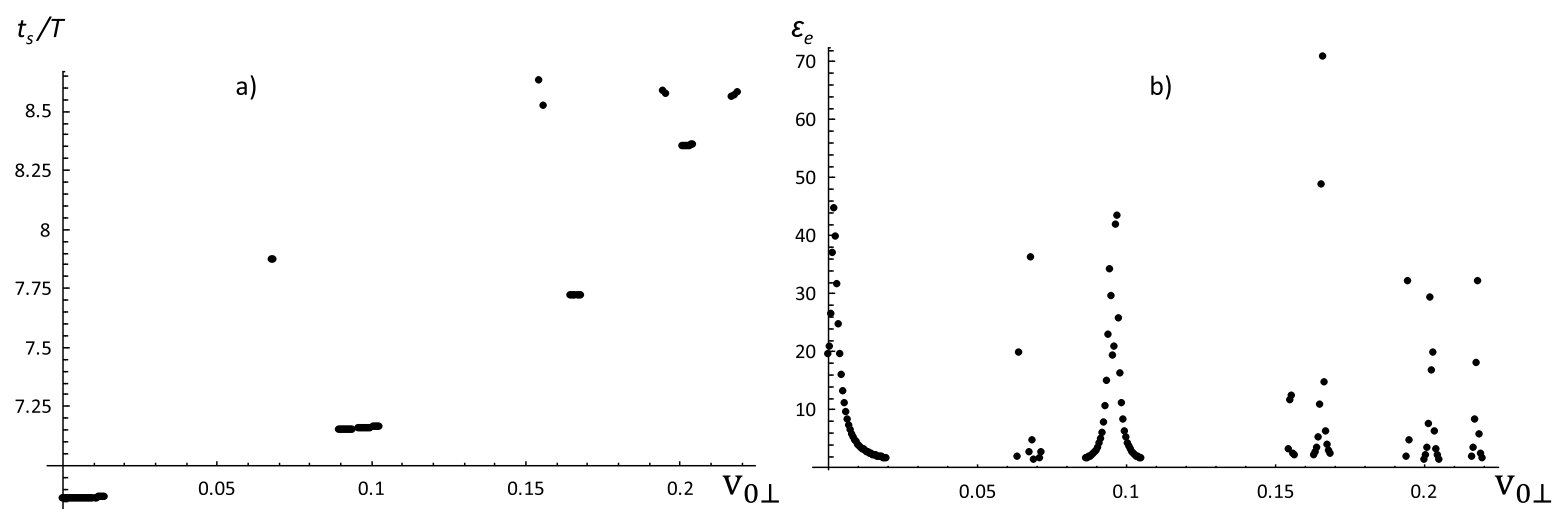

Fig. 4. Dependence of the recollision characteristics in a series of 500 trials with the initial electron perpendicular velocity varying from 0 to 0.25 a.u.: (a) collision time in units of laser period; (b) electron energy at the collision time in a.u. The laser parameters are the same as in Figure $3 \mathrm{~b}$.

The excitation probability for a given launch time $t_{0}$ can be introduced as an average over the initial distribution of electrons over the transverse velocities:

$$
\begin{aligned}
w_{B X}\left(t_{0}\right)= & \int_{0}^{v_{\max \perp}} d v_{\perp} v_{\perp} p_{v}\left(v_{\perp}\right) H\left[r_{B X}\left(\varepsilon_{e}\left(t_{s}\right)\right)-r_{e}\left(t_{s}\right)\right] \\
& \times H\left[\varepsilon_{e}\left(t_{s}\right)-\varepsilon_{B X}\right]
\end{aligned}
$$

where $p_{v}$ is the initial electron distribution function defined by equation (3). The two Heaviside functions select the electrons verifying the ion excitation conditions. The convergence of the averaging procedure has been verified by increasing the number of trail electrons $N_{v}$, so that the excitation probability was calculated with a precision better than a few percent.

Typically, the probability of excitation $w_{B X}$ is of a few percent because of the divergence of the electron cloud. This value can be crudely estimated as a ratio of the excitation cross section (1) to the cross section of the electron cloud returning to the parent ion. The maximum excitation cross section is $\sim 10$ a.u. corresponding to a radius of interaction $\sim 2$ a.u. It has to be compared to the characteristic radius of an electron cloud after one laser period $\sim\left\langle v_{\perp}\right\rangle T \simeq 0.2 \times 100 \simeq 20$ a.u. Thus the typical cross section of the electron cloud at the time of the first recollision is $\sim 1000$ a.u., which is hundred times larger than the excitation cross section. This crude estimate corresponds to the one proposed in reference [26]. However, the recollisions at subsequent electron returns increase the probability of ion excitation by a factor of $2-3$. Thus recollisions are indeed important for the proper evaluation of the total number of excited ions.

A similar approach can be used to calculate the average dipole momentum of excited ions, $\boldsymbol{d}_{B}=\left\langle-e \boldsymbol{r}_{B}\right\rangle$, where $\boldsymbol{r}_{B}$ is the $\mathrm{B}^{2} \Sigma_{u}^{+}$electron orbital. The absolute value of the dipole momentum for the $\mathrm{B} \rightarrow \mathrm{X}$ transition is known, $d_{B X} \simeq 0.7$ a.u. [27], and its direction and phase are defined by the recollision conditions. It follows from the symmetry of interaction that only the component of momentum parallel to the electric field direction is non-zero. Then the average dipolar momentum for $t>t_{s}$ can be written as $d_{B X} p_{B X}\left(t_{0}\right) \exp \left(-i \omega_{B X} t\right)$, where $\omega_{B X}=\varepsilon_{B X} / \hbar$ and the probability $p_{B X}\left(t_{0}\right)$ for the launch time $t_{0}$ is calculated as follows:

$$
\begin{aligned}
p_{B X}\left(t_{0}\right)= & \int_{0}^{v_{\max \perp}} d v_{\perp} v_{\perp} p_{v}\left(v_{\perp}\right) H\left[r_{B X}\left(\varepsilon_{e}\left(t_{s}\right)\right)-r_{e}\left(t_{s}\right)\right] \\
& \times H\left[\varepsilon_{e}\left(t_{s}\right)-\varepsilon_{B X}\right] \cos \theta_{s} \mathrm{e}^{-i \omega_{B X} t_{s}} .
\end{aligned}
$$

Here, $\theta_{s}$ is the angle of momentum transferred to the ion in the recollision process with respect to the $x$-axis. It is defined as a difference between the moments of the electron approaching and leaving the ion at a distance $r_{e} \simeq r_{B X}$. The last factor in equation (10) accounts for the recollision phase. In fact, for two subsequent half-periods, the electrons born at times $t_{0}$ and $t_{0}+\frac{1}{2} T$ have approximately the same ionization and recollision probabilities, but they approach the parent ion from opposite directions. So the contributions to the dipole excitation probability from two subsequent periods partially cancel out. In particular, the dipole excitation probability is exactly zero if the laser frequency is equal to a half of the transition frequency, $\omega_{0}=\frac{1}{2} \omega_{B X}$ and the laser pulse duration is equal to an integer number of periods. This is a consequence of the symmetry of the electronic states of the nitrogen ion and the destructive interference between the $\mathrm{B}$ and $\mathrm{X}$ states. This feature has been observed in paper [14] and leads to a variation of the lasing intensity with pump pulse duration and wavelength, thus providing a link of the recollision model to experimental results.

\subsection{Scan over the ionization time}

The excitation probability per each half laser period $k$ is defined as

$$
W_{k B X}=\int_{k T / 2}^{(k+1) T / 2} d t_{0} w_{B X}\left(t_{0}\right) w_{\text {ion }}\left(t_{0}\right) / W_{\text {ion }} .
$$

For the sake of convenience, it is normalized to the total ionization probability. Thus the total fraction of ions in the excited state $W_{B X}=\sum_{1}^{M} W_{k B X}$ is evaluated as a sum of the excitation probabilities $W_{k B X}$ over all laser 

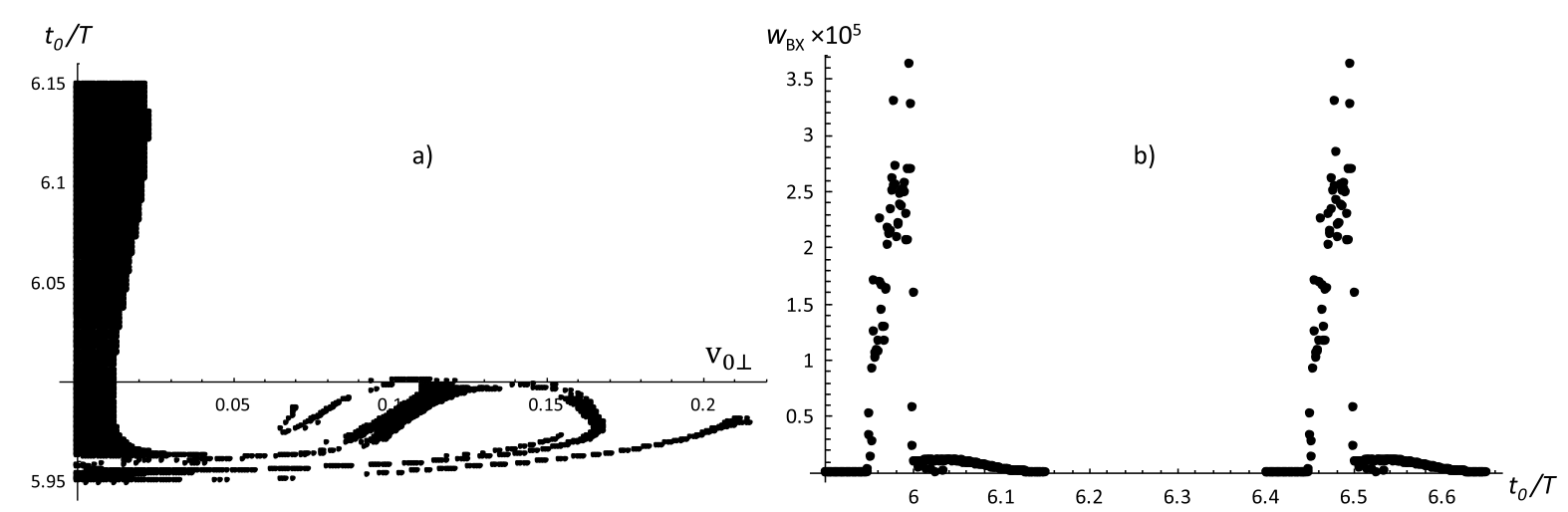

Fig. 5. (a) Domains in the plane $v_{0 \perp}, t_{0}$ contributing to the recollision process during a half of laser period. (b) Dependence of the ion excitation probability on the electron launch time $t_{0}$ within one laser period. The laser parameters are the same as in Figure 3.

half-periods $M$. A similar approach is applied for the calculation of the average dipole excitation probability (10) for each half laser period:

$$
P_{k B X}=\int_{k T / 2}^{(k+1) T / 2} d t_{0} p_{B X}\left(t_{0}\right) w_{\text {ion }}\left(t_{0}\right) / W_{\text {ion }}
$$

The total dipole excitation probability is calculated then as $P_{B X}=\sum_{1}^{M} P_{k B X}$.

Figure 5a shows the domains in the plane $v_{0 \perp}, t_{0}$ contributing to the recollision process. Two zones can be readily identified. A zone of small transverse initial velocities, $v_{0 \perp}<0.03$, where all orbits are returning to the parent ion, and a "parachute" zone of large transverse velocities $v_{0 \perp}<0.2$, where some trajectories returning to the origin because of the Coulomb attraction of the parent ion. This zone is highly structured and exist for negative launch times (before the field maximum) only. Even though the size of the second zone is relatively small it makes a significant contribution to the recollision probability because of a larger phase volume.

Figure 5b shows that contributions of two consecutive half periods corresponding to the positive and negative electric fields are very much similar. The excitation probability fluctuates strongly as a function of the electron release time for early electron launches. This is explained by a complex structure of the phase space shown in panel (a). The later launch times show a more regular behavior, but the absolute value of the excitation probability is much smaller. Strong fluctuations of the excitation probability are also partially due to our assumption of a sharp cutoff of the excitation cross section. A smoother interpolation of the excitation probability would reduce the fluctuations, but will not affect the final result.

Figure 6 presents a time dependence of the ion excitation probability $W_{k B X}$ and of the dipolar excitation probability $P_{k B X}$ for three laser intensities. The excitation probability shows a bell-shaped form attaining its maximum at the maximum of laser intensity. It decreases monotonously with laser intensity. This decreasing trend can be understood as follows: the ionization probability is an exponential function of the laser intensity and thus it increases faster than the recollision excitation probability, which depends weakly on the laser field. So, the number of excited ions increases with the intensity, but slower than the total number of ions.

The dipolar excitation probability changes sign each half laser period similarly to the laser electric field. So the contributions from subsequent half periods partially cancel out. An incomplete canceling is explained by a difference between the laser and dipole periods. For this reason the imaginary part changes sign in the middle of the pulse in the example shown in panel (c).

\subsection{Simulation results}

We studied the dependence of the fraction of excited molecules and of their dipolar moment on the laser wavelength, intensity, pulse duration and chirp. Following the conditions of experiment [14], the laser wavelength is varied from 810 to $770 \mathrm{~nm}$, the laser intensity - from 1 to $2 \times 10^{14} \mathrm{~W} / \mathrm{cm}^{2}$, the pulse duration $t_{\text {las }}-$ from 15 to $50 \mathrm{fs}$, and the chirp $C$ - in the range from -1 to 1 . The excitation probability $W_{B X}$ and the average dipole moment $P_{B X}$ are calculated according to equations (11) and (12) and summed over subsequent semiperiods.

The dependencies of the ionization probability and of the excitation probability on the laser intensity are shown in Figure 7a. The ionization probability $W_{\text {ion }}$ (violet line) is an exponential function of the laser intensity and it increases from $0.3 \%$ to $10 \%$ when the laser intensity increases from 1 to $2 \times 10^{14} \mathrm{~W} / \mathrm{cm}^{2}$. In contrast, the recollision excitation probability $W_{B X \text { rec }}$ (green line) is a weak function of the laser intensity. It decreases from $2.4 \%$ to $1.5 \%$ in the same laser intensity interval. It is important to compare the number of excited ions created in recollisions with the direct laser ionization in the excited state $W_{B X \text { dir }}$, which is shown in panel (a) with a dark blue line. The trends of two lines are opposite: while the direct ionization probability increases, the probability of recollision excitation decreases. Thus the recollision process is important for laser intensities lower than $2 \times 10^{14} \mathrm{~W} / \mathrm{cm}^{2}$. The total recollision probability $W_{B X \text { tot }}$ is almost constant in that laser intensity interval (light blue line). It amounts to $\sim 3.5 \%$ in that case. 

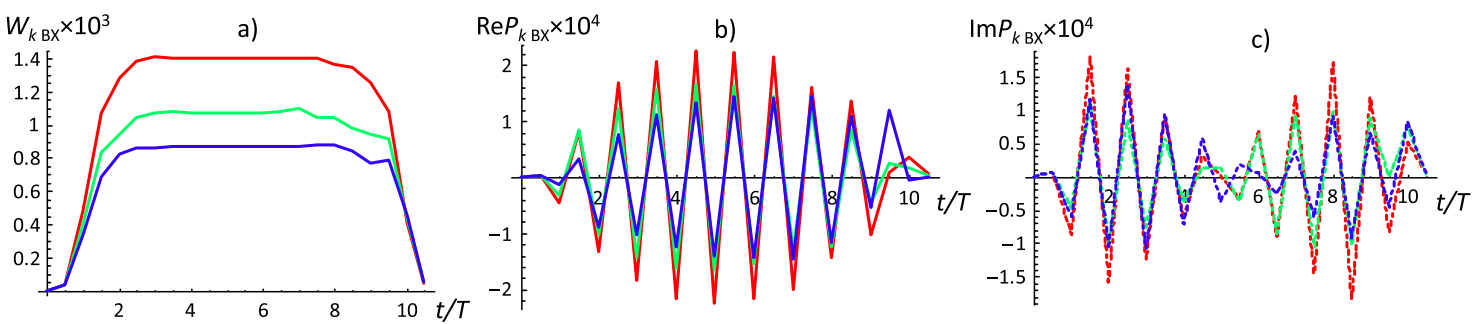

Fig. 6. (a) Excitation probability $W_{k B X}$ for a half of laser period in function of time (solid lines). Real (b) and imaginary (c) parts of the dipolar excitation probability in function of time. Laser parameters are: intensity 1 (red), 1.5 (green) and $2 \times 10^{14} \mathrm{~W} / \mathrm{cm}^{2}$ (blue), wavelength $800 \mathrm{~nm}$, pulse duration $30 \mathrm{fs}$ and zero chirp. Points corresponding to each half period are connected by lines for better visibility.
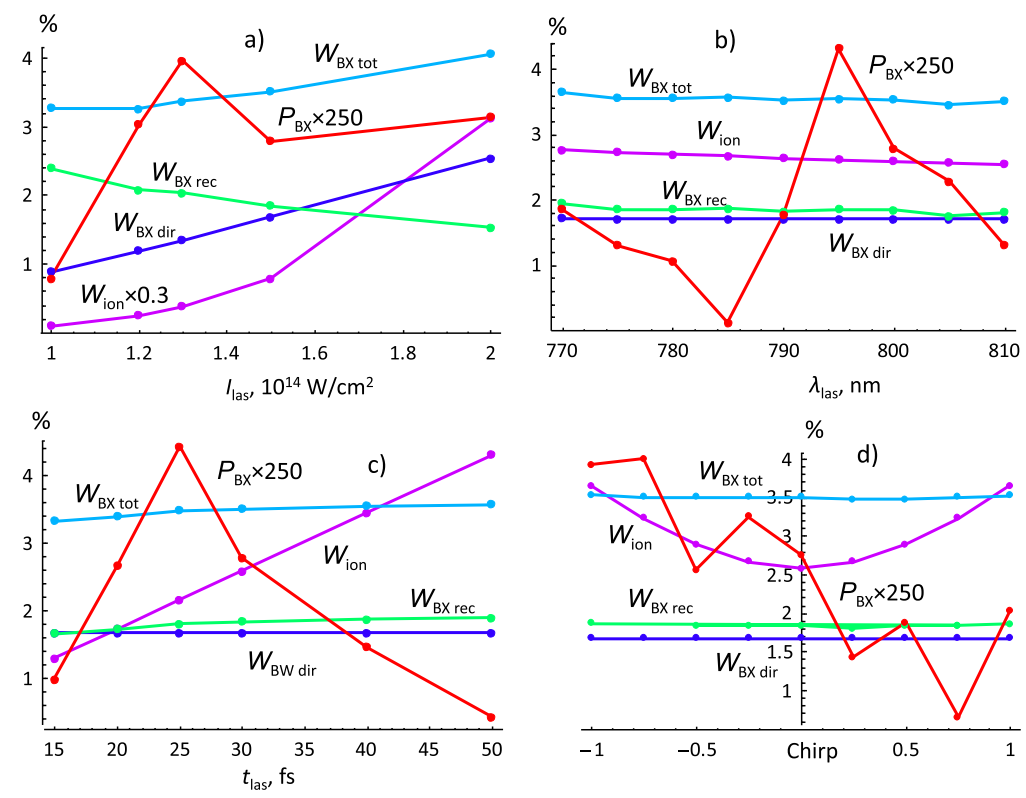

Fig. 7. Dependence of the recollision excitation probability ( $W_{B X \text { rec green line), direct ionization-excitation probability ( } W_{B X} \text { dir }}$ blue line) and the ionization probability ( $W_{\text {ion }}$ violet) on the laser parameters. Light blue line shows the total excitation probability $W_{B X \text { tot }}$ and red line shows the dipolar excitation probability $P_{B X}$. (a) Dependence on the laser intensity for the laser wavelength $800 \mathrm{~nm}$ and pulse duration $30 \mathrm{fs}$. (b) Dependence on the laser wavelength for the laser intensity $1.5 \times 10^{14} \mathrm{~W} / \mathrm{cm}^{2}$ and pulse duration $30 \mathrm{fs}$. (c) Dependence on the laser pulse duration for the wavelength $800 \mathrm{~nm}$ and laser intensity $1.5 \times 10^{14} \mathrm{~W} / \mathrm{cm}^{2}$. (d) Dependence on the laser chirp $C$ for the laser intensity $1.5 \times 10^{14} \mathrm{~W} / \mathrm{cm}^{2}$, wavelength $800 \mathrm{~nm}$ and pulse duration $30 \mathrm{fs}$. Points show the simulation results, the lines are traced for better visibility.

The dependencies of the recollision excitation probability and of the ionization probability on the laser wavelength are shown in Figure 7b. Both of them decrease slightly with the laser wavelength similar to the ionization probability. Such a trend can be explained by the fact that a pulse of same duration contains a smaller number of laser periods and consequently smaller ionization and recollision probabilities. The dependencies of the ionization and excitation probability on the laser pulse duration are shown in Figure 7c. While the ionization probability increases linearly with the laser pulse duration, the relative number of excited ions remains the same. Thus the pulse duration does not affect the excitation probability, while the total number of excited ions increases linearly with the laser pulse duration.
A similar conclusion applies to the dependence on the laser pulse chirp shown in Figure 7d. There is no dependence of the ionization and excitation probabilities on the chirp sign, but the ionization probability increases with the chirp, because larger $C$ corresponds to a longer pulse duration $t_{\text {las }}=t_{0 \text { las }}\left(C^{2}+1\right)^{1 / 2}$. Conversely, the excitation probability remains approximately the same, similarly to its dependence on the laser pulse duration with zero chirp as shown in panel (c). For a wavelength of $800 \mathrm{~nm}$ and an intensity $1.5 \times 10^{14} \mathrm{~W} / \mathrm{cm}^{2}$ the runs with chirps $C= \pm 1$ show approximately the same total excitation probability of $3.5 \%$ as in the case of a zero chirp, while the ionization probability increases from $2.6 \%$ to $3.7 \%$. These results show that recollisions make a significant contribution to the creation of molecular ions in excited states near 
the ionization threshold, but it is a nonresonant process, which depends weakly on the laser pulse parameters.

We also studied the dependence of the average dipolar moment of the ion in the excited state $P_{B X}$ on the laser parameters. As the electrons in the subsequent laser half periods approach the parent ion from opposite directions, their contributions partially cancel out, and the average dipolar moment excitation probability is approximately 200 times smaller than the excitation probability. However, as the interference of the contributions from the subsequent half cycles depends on the frequency detuning $\Delta \omega=\omega_{0}-\frac{1}{2} \omega_{B X}$, the average dipolar moment shows characteristic variations in function of the laser parameters. This effect is discussed in reference [14] experimentally and with a simplified one-dimensional recollision model. Our three-dimensional model confirms their results. In the case of exact resonance, $\Delta \omega=0$, the average dipolar moment is close to zero, which manifests itself as a very small emission signal. This can be seen in Figure $7 \mathrm{~b}$ (red line) as a minimum at the wavelength of $785 \mathrm{~nm}$. Another minimum is found at a larger wavelength of $810 \mathrm{~nm}$ for a pulse duration of $30 \mathrm{fs}$. It corresponds to the condition of destructive interference reached when $\Delta \omega \simeq$ $\pi / t_{\text {olas }}$ in agreement with the experimental result [14].

A non-monotonous dependence of the dipolar moment excitation probability on the laser pulse duration is demonstrated in Figure 7c. The laser pulse duration of $25 \mathrm{fs}$ corresponds to the constructive interference of dipolar moments. Shortening or increasing the pulse duration results in a smaller value of the dipolar moment, which is directly related to the amplitude of the amplified signal. The dependence of the dipolar moment on laser intensity is also non-monotonous. It increases with laser intensity for $I_{\text {las }}<1.3 \times 10^{14} \mathrm{~W} / \mathrm{cm}^{2}$ and then slightly decreases. Small variations of $P_{B X}$ on the order of $20-30 \%$ are within the calculation precision. The cancellation of the dominant terms when the contributions from subsequent half periods are added significantly reduces the accuracy of the calculation of $P_{B X}$, which depends also on the exact laser pulse profile. The dipolar moment shows an evident decreasing trend as a function of the laser chirp. This feature merits to be verified in experiments.

\section{Conclusion}

Our analysis suggests that for typical interaction conditions about $1-10 \%$ of the nitrogen gas molecules could be ionized with the laser pulse. The liberated electrons can enter in the recollision with parent ions. Modeling of the quantum divergence of electron cloud moving in the laser field with multiple electron trajectories shows that about $1-2 \%$ of these molecules could be effectively excited due to the electron recollisions. This estimate agrees well with the one proposed in the simplified one-dimensional model [5]. One should, however, note that our quasi-classical model cannot account for phase correlations in ion excitation in subsequent electron collisions. As the electron collisions with the same ion are correlated by the laser field temporal evolution, two subsequent recollisions may induce an interference between the ground and excited ionic states for each molecule. This issue requires a special quantum-mechanical analysis. However, our simplified method of calculation of the average dipolar moment shows a good agreement with the experiment in what concerns its dependence on the laser pulse duration and wavelength.

Considering the density of nitrogen molecules of $2 \times$ $10^{19} \mathrm{~cm}^{-3}$ in air at normal pressure, we expect the density of ionized molecules to be on the order of $(2$ $3) \times 10^{17} \mathrm{~cm}^{-3}$. Having an excitation probability of $3.5 \%$, we may expect a density of excited nitrogen ions at the level of $10^{16} \mathrm{~cm}^{-3}$. For a filament radius of $\sim 50 \mu \mathrm{m}$ and a length of $2-4 \mathrm{~cm}$, the total number of excited ions would be more than $10^{12}$. Having the energy of each photon of $\sim 3 \mathrm{eV}$, the total energy stored in the excited state in one filament would be about $0.5 \mu \mathrm{J}$. There were about 20 filaments created in the experiment [4], and the total energy of the amplified signal was $2.6 \mu \mathrm{J}$ at the wavelength of $428 \mathrm{~nm}$. Thus a relatively small fraction of less than $5 \%$ of nitrogen molecules brought in the excited ionic state $\mathrm{B}^{2} \Sigma_{u}^{+}$is sufficient to account for the energy of the amplified signal pulse. This fraction of excited ions cannot be explained by a direct laser excitation, but it is consistent with the excitation by the recollision process. We emphasize, however, that no population inversion can be realized by the recollisions.

The developed approach can be easily extended to include other competing inelastic processes, the laser pulse polarization, etc.

This work was partially accomplished as an internship project of Jean-Félix Tremblay-Bugeaud at CELIA. The authors acknowledge the financial support for the internship from the French National Research Agency (ANR) in the framework of "the investments for the future" Programme IdEx BordeauxLAPHIA (ANR-10-IDEX-03-02). Yi Lui acknowledge the support of National Natural Science Foundation of China (Grant No. 11574213), Innovative Program of Shanghai Municipal Education Commission (Grant No. 2017-01-07-00-07-E00007), and the Program for Professor of Special Appointment (Eastern Scholar) at Shanghai Institutions of Higher Learning (No. TP2014046).

\section{Author contribution statement}

V.T.T., Y.L. and A.M. put the main hypothesis and the general framework. J.F.T.B. and V.T.T. wrote the programs and performed the calculations. All the authors contributed to discussions and in the preparation of the manuscript.

\section{References}

1. J. Yao, B. Zeng, H. Xu, G. Li, W. Chu, J. Ni, H. Zhang, S.L. Chin, Y. Cheng, Z. Xu, Phys. Rev. A 84, 051802(R) (2011)

2. J. Ni, W. Chu, C. Jing, H. Zhang, B. Zeng, J. Yao, G. Li, H. Xie, C. Zhang, H. Xu, S.L. Chin, Y. Cheng, Z. Xu, Opt. Express 21, 22791 (2013) 
3. W. Chu, G. Li, H. Xie, J. Ni, J. Yao, B. Zeng, H. Zhang, C. Jing, H. Xu, Y. Cheng, Z. Xu, Laser Phys. Lett. 11, 01301 (2014)

4. G. Point, Y. Liu, Y. Brelet, S. Mitryukovskiy, P.J. Ding, A. Houard, A. Mysyrowicz, Opt. Lett. 39, 1728 (2014)

5. Y. Liu, P. Ding, G. Lambert, A. Houard, V.T. Tikhonchuk, A. Mysyrowicz, Phys. Rev. Lett. 115, 133203 (2015)

6. J. Yao, S. Jiang, W. Chu, B. Zeng, C. Wu, R. Lu, Z. Li, H. Xie, G. Li, C. Yu, Z. Wang, H. Jiang, Q. Gong, Y. Cheng, Phys. Rev. Lett. 116, 143007 (2016)

7. P.B. Corkum, Phys. Rev. Lett. 71, 1994 (1993)

8. J.L. Krause, K.J. Schafer, K.C. Kulander, Phys. Rev. Lett. 68, 3535 (1992)

9. V.R. Bhardwaj, D.M. Rayner, D.M. Villeneuve, P.B. Corkum, Phys. Rev. Lett. 87, 253003 (2001)

10. G.M. Fraiman, V.A. Mironov, A.A. Balakin, Phys. Rev. Lett. 82, 319 (1999)

11. A. Brantov, W. Rozmus, R. Sydora, C.E. Capjack, V.Yu. Bychenkov, V.T. Tikhonchuk, Phys. Plasmas 10, 3385 (2003)

12. G. Rascol, H. Bachau, V.T. Tikhonchuk, H.-J. Kull, T. Ristow, Phys. Plasmas 13, 103108 (2006)

13. T. Brabec, M.Yu. Ivanov, P.B. Corkum, Phys. Rev. A 54, R2551 (1996)

14. Y. Liu, P. Ding, N. Ibrakovic, S. Bengtsson, S. Chen, R. Danylo, E.W. Simpson, E.W. Larsen, C. Arnold, A. Houard, J. Mauritsson, A. L'Huillier, S. Zhuang, V. Tikhonchuk, A. Mysyrowicz, Phys. Rev. Lett. (submitted)
15. D.H. Crandall, W.E. Kauppila, R.A. Phancuf, P.O. Taylor, G.H. Dunn, Phys. Rev. A 9, 2545 (1974)

16. X. Sun, S. Xu, J. Zhao, W. Liu, Y. Cheng, Z. $\mathrm{Xu}$, S.L. Chin, G. Mu, Opt. Express 20, 4790 (2012)

17. S.I. Mitryukovskiy, Y. Liu, A. Houard, A. Mysyrowicz, J. Phys. B: At. Mol. Opt. Phys. 48, 094003 (2015)

18. F. Cloux, B. Fabre, B. Pons, Phys. Rev. A 91, 023415 (2015)

19. J. Muth-Böhm, A. Becker, F.H.M. Faisal, Phys. Rev. Lett. 85, $2280(2000)$

20. A.M. Perelomov, V.S. Popov, M.V. Terent'ev, Sov. Phys. JETP 23, 924 (1966)

21. V.S. Popov, Phys. Uspekhi 47, 855 (2004)

22. A. Talebpour, S. Larochelle, S.L. Chin, J. Phys. B 31, L49 (1998)

23. T.K. Kjeldsen, L.B. Madsen, Phys. Rev. A 71, 023411 (2005)

24. S.-F. Zhao, L. Liu, X.-X. Zhou, Opt. Commun. 313, 74 (2014)

25. A. Lofthus, P.H. Krupenie, J. Phys. Chem. Ref. Data 6, 113 (1977)

26. H. Xu, E. Lötstedt, A. Iwasaki, K. Yamanouchi, Nat. Commun. 6, 8347 (2015)

27. S.R. Langhoff, Ch.W. Bauschglicher Jr., J. Chem. Phys. 88, 329 (1988) 